Rev. Téc. Ing. Univ. Zulia. Vol. 42, No. 2, 2019, 57-66

\title{
Analysis of Multistage Pulsed Current Drivers for Solid State Lighting Devices
}

\author{
C. Alvarez $\mathbb{D}$ \\ Prof. Jubilado, Escuela de Ingeniería Eléctrica, Facultad de Ingeniería, Universidad del Zulia, Maracaibo, C.P. 4001, \\ Venezuela. \\ cealvarez@fing.luz.edu.ve \\ https://doi.org/10.22209/rt.v42n2a02
}

Recepción: 12/06/2018 | Aceptación: 07/01/2019 | Publicación: 01/05/2019

\begin{abstract}
This article analyzes, through simulations, high brightness LED multistage pulsed current drivers used in solid state lighting. These drivers consist of a full wave rectifier with 1 to 8 stages of pulsed current. As the circuits do not use capacitors and inductors, they have advantages over the switched power supplies, such as: simplicity, low cost, long life, high power factor, surge protection and the possibility to be integrated on a chip; for the other hand, these drivers do not produce electromagnetic interference. The methodology consists of modeling the power supply and LEDs or chips of LED (COB-LEDs), which are switched according the changes in the line voltage. The results show that by using a number of stages of constant current between 5 and 8, efficiencies between $86 \%$ and $91 \%$ are obtained, power factor is maintained above 0,996, Total Harmonic Distortion is below $9 \%$ and it complies with the IEC 61000-3-2 class C standard, which refers to the lighting equipment maximum percentage of harmonics.
\end{abstract}

Keywords: LED Lighting, LED drivers, power factor, IEC 61000.

\section{Análisis de Fuentes de Alimentación Multietapas de Corriente Pulsante para Dispositivos de Iluminación de Estado Sólido}

\begin{abstract}
Resumen
En este artículo se analiza, por medio de simulaciones, fuentes de alimentación multietapas de corriente pulsante para leds de alto brillo utilizados en iluminación de estado sólido. Estas fuentes consisten en un rectificador de onda completa con 1 a 8 etapas de corriente pulsante. Como los circuitos no utilizan condensadores ni inductores, presentan ventajas con respecto a las fuentes conmutadas, tales como: simplicidad, bajo costo, larga vida útil, alto factor de potencia, protección contra sobretensiones y posibilidad de ser integrado en un chip; además no produce interferencia electromagnética. La metodología utilizada consiste en modelar la fuente de alimentación y los leds o chips de leds (COB-LEDs), los cuales se conmutan de acuerdo a los cambios del voltaje de línea. Los resultados muestran que al utilizar un número de etapas de corriente constante entre 5 y 8, se obtienen eficiencias entre el 86\% y 91\%, los factores de potencia se mantienen por encima de 0,996, la distorsión armónica total está por debajo del 9\% y se cumple con la norma IEC 61000-3-2, clase C, la cual está referida al contenido máximo porcentual de armónicos en equipos de iluminación.
\end{abstract}

Palabras clave: Iluminación led, fuentes de alimentación led, factor de potencia, IEC 61000. 


\section{Introducción}

Los leds blancos de alto brillo han ido desarrollándose y presentándose como una opción de eficiencia energética para la iluminación en general. Las ventajas de esta tecnología son evidentes: alta eficacia, largo tiempo de vida útil, tamaño reducido, diversidad de colores, entre otras [1 - 4]. Esto da a los leds un gran número de aplicaciones, tales como: iluminación interior y exterior, pantallas de televisión, automoción, luces decorativas, semáforos, etc.

Por lo general, las fuentes de alimentación para leds en la iluminación de estado sólido, consisten en un circuito que produce una corriente continua constante que está alimentada por un rectificador y una fuente conmutada para la conversión DC a DC. Este tipo de fuente, es común que tenga un bajo factor de potencia, genere armónicos en la corriente de entrada y produzca interferencia electromagnética [5]. Por otro lado, como las fuentes conmutadas utilizan condensadores electrolíticos, su funcionamiento es susceptible a que ocurran fallas, por lo que suelen dañarse mucho antes de acabar la vida útil de los leds que alimentan [6].

Otro tipo de circuito de alimentación para la iluminación de estado sólido, consiste en fuentes rectificadas que entregan tensión a un número determinado de leds con una resistencia en serie, los cuales son denominados AC-LEDs [7]. Este tipo de circuito ha sido criticado porque produce efectos de parpadeo (flicker). Para solucionar este problema, se le puede colocar un condensador electrolítico después del rectificador, el cual, estabiliza la corriente del arreglo de leds. Sin embargo, este condensador reduce más el factor de potencia, aumenta la distorsión armónica de la corriente de entrada y acorta la vida de la fuente de alimentación.

Hay otros esquemas de fuentes de alimentación, uno de ellos consiste en la no utilización de inductores ni condensadores, lo que significa que se reduce su costo, y su vida útil es ilimitada $[6,8,9]$. Esta fuente de alimentación está formada por varias etapas que producen pulsos de corriente constante, las cuales conmutan los grupos de arreglos de leds o chips de leds (COB-LED) estratégicamente ubicados, de manera que, la corriente del circuito se asemeja a la de una señal sinusoidal, ver figura 1. Como en el circuito se aprovecha mejor el voltaje de entrada, entonces se presentan características muy favorables: reduce el índice de parpadeo, aumenta el factor de potencia, mejora la eficiencia, reduce la distorsión armónica en la corriente de entrada, elimina las emisiones electromagnéticas y no usa condensadores.
Gráfico Intensidad vs. Ángulo en fuentes de corriente pulsante de una y cuatro etapas.
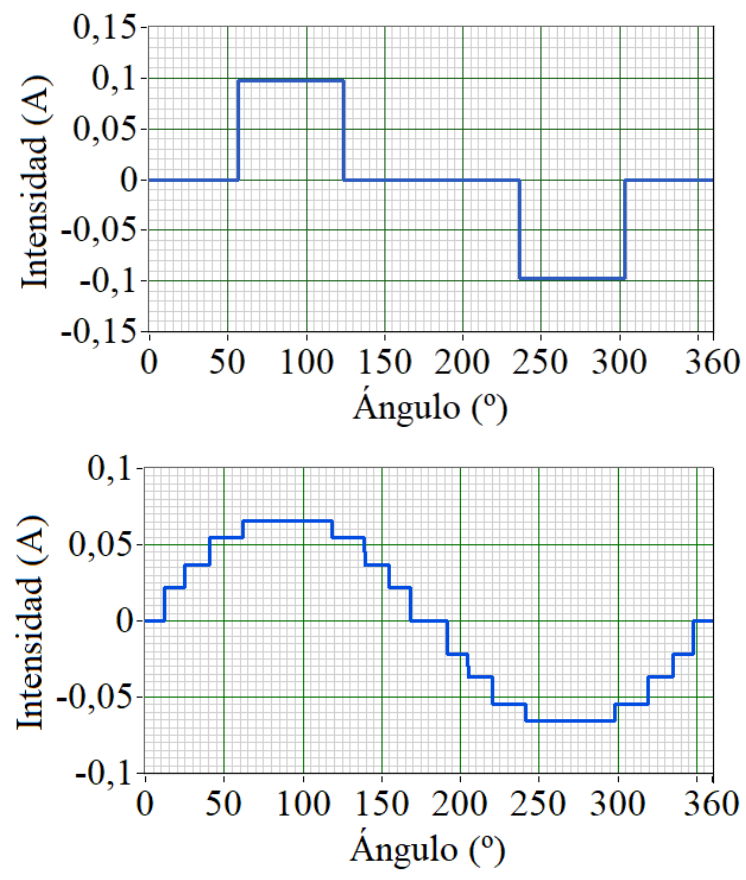

Figura 1. Formas de ondas de intensidad en fuentes de corriente pulsante para leds de una y cuatro etapas.

En este trabajo, se hace un análisis de este último tipo de fuente de alimentación y su evaluación, mediante simulaciones de los modelos matemáticos de los circuitos, para los casos de 1 a 8 etapas. De esta manera se pude hacer comparaciones de: eficiencia, factor de potencia, estabilidad en la potencia de salida y contenido de armónicos en las corrientes de entrada.

\section{Desarrollo de las Ecuaciones de las Fuentes de Alimentación para Leds}

La curva característica de un arreglo de leds, se puede aproximar a dos líneas rectas equivalentes a una fuente de voltaje constante con una resistencia serie [3]. Sin embargo, se puede obtener un modelo más exacto, por medio de una aproximación polinómica de segundo orden, la cual se va a utilizar para los análisis de los circuitos de este trabajo. El mecanismo de control de las fuentes de corriente de cada etapa, ocurre al haber una resistencia que detecta la corriente de un transistor MOSFET, que puede ser realimentado en la compuerta por medio de un transistor bipolar o un amplificador operacional [8, 5], ver figura 2. 
La fuente de alimentación de corrientes pulsantes, propuesta en este trabajo, es mostrada en la figura 2, la cual presenta un circuito de conmutación automática de cuatro etapas. Para empezar el análisis, se considera que los leds representados en el circuito (LED1, LED2, LED3 y LED4) son COB-LEDs o arreglos de leds. En el momento inicial, cuando la tensión de línea es igual a 0 V, los transistores Q1, Q4, Q7 y Q10 están en estado de corte. Luego, la tensión rectificada se eleva, el voltaje entre compuerta y fuente $\left(V_{G S 3}\right)$ de $Q 3$ se incrementa en la misma proporción, al igual que en los terminales ánodo y cátodo del $L E D 1$. La corriente $I_{D S 3}$ de $Q 3$ es nula, hasta que comienza a conducir el LED1; en ese momento empieza a subir la $I_{D S 3}$ y la tensión en $R 4$. Cuando el voltaje en $R 4$ polariza la base de $Q 2$, alrededor de $0,66 \mathrm{~V}$, se reduce la tensión de su colector, que a su vez alimenta la compuerta de $Q 3$ y comienza a detener el incremento de $I_{D S 3}$; en otras palabras, se produce una realimentación de la tensión en esa compuerta hasta conseguir un equilibrio cuando la corriente $I_{D S 3}$ en $Q 3$ es aproximadamente $0,66 \mathrm{~V} / R 4$, que es el valor de la intensidad de la primera fuente de corriente del grupo y que enciende al LED1. Los valores de intensidad de las otras fuentes de corriente se fijan por medio de las resistencias de fuente $R s(R 8, R 12, \mathrm{y} R 16)$, respectivamente y los voltajes base emisor de $Q 5, Q 8$ y Q11. El voltaje base emisor de los transistores bipolares tiene un coeficiente negativo de temperatura, lo cual es útil para reducir las corrientes de las etapas en caso de calentamiento. mas el $L E D 2$. En este momento, $Q 6$ se enciende y actúa la segunda fuente de corriente, que alimenta a LED1 y LED2 en serie ya que se desconecta Q3. Para los otros grupos de fuente de corriente, se producen los mismos procesos mientras aumenta la tensión rectificada. Un orden inverso de conmutación de las fuentes de corrientes ocurre al disminuir la tensión rectificada. Para obtener una forma de onda de corriente similar a la sinusoidal, se deben seleccionar los valores adecuados de las resistencias de fuente Rs.

El circuito de la figura 2, además ofrece una protección contra sobretensiones, ya que, si se eleva la tensión rectificada, el divisor de tensión formado por $R 13$ y $R 14$ apaga a $Q 12$ y, con todos los demás MOSFETs apagados, el circuito estará protegido hasta un valor de tensión de línea igual al voltaje máximo de drenaje-fuente especificado para estos MOSFETs y el voltaje máximo pico inverso del puente rectificador. En otras palabras, el circuito no deja de funcionar, pero cuando hay una sobretensión, se apagan los MOSFETs y se reduce el consumo de potencia de la fuente de alimentación. De todas formas, es recomendable colocar una protección adicional formada por un fusible y un varistor en la entrada del circuito.

En estas fuentes de alimentación, para poder reducir la distorsión de la corriente de entrada, es necesario aumentar el número de etapas de fuentes de

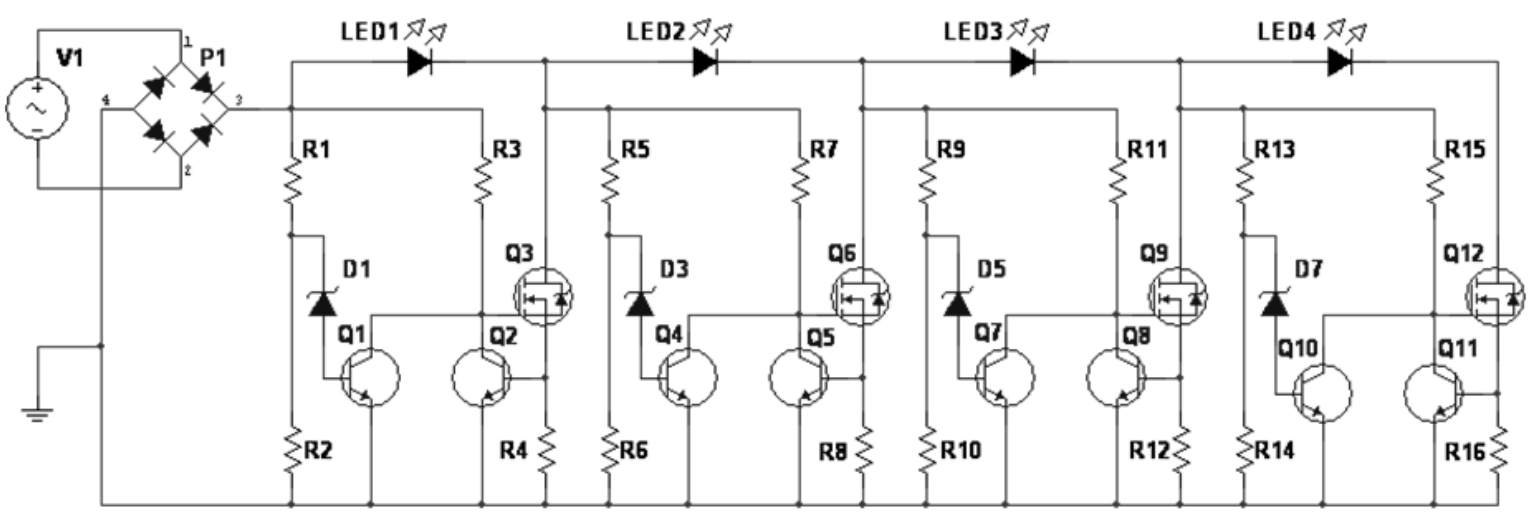

Figura 2. Fuente de alimentación de corriente pulsante de 4 etapas para un arreglo de leds.

La primera fuente de corriente se apaga cuando la tensión del divisor formado por $R 1$ y $R 2$ alcanza la suma del voltaje del diodo Zener $D 1$ y el de la base emisor de $Q 1$, por lo que este se satura, reduce el voltaje $V_{G S 3}$ a casi $0 \mathrm{~V}$ y lo apaga. Se utilizan Zeners de 6,2 V, ya que su coeficiente de variación de temperatura es similar al de la unión base emisor, pero con signo contrario. El divisor de tensión se calcula para apagar a Q3 cuando el voltaje rectificado es la suma de los voltajes del LED1 corriente constante. Los valores de las corrientes de cada etapa, $I_{C S n}$, que fluyen a través de los arreglos de los leds pueden ser determinados por medio de la siguiente ecuación:

$$
I_{C S n}=\frac{V_{B E(3 n-1)}}{R_{4 n}}
$$

Donde $\quad n=1,2,3 \ldots$ hasta el máximo número de etapas de fuentes de corrientes $m$. 
$V_{B E} \approx 0,66 V$ son los voltajes de activación de base-emisor de Q2, Q5, Q7...

$R 4, R 8, R 12 \ldots$ son las resistencias de detección de corriente de cada etapa.

Para una fase igual a 0, la expresión del voltaje de línea está dada por la ecuación 2:

$$
V_{\text {linea }}(t)=V_{P} \operatorname{sen}(\omega t)
$$

Donde $\quad V p$ es el voltaje pico de línea.

$\omega$ es la frecuencia angular $(2 \pi f)$.

$t$ es el tiempo.

El producto $\omega t$ es igual a $\theta$, que es un ángulo que cambia en función del tiempo. El voltaje eficaz rectificado se halla según la ecuación 3:

$$
V_{\text {RMS_Rect }} \approx \frac{V_{P_{-} \text {Rect }}}{\sqrt{2}}
$$

Donde: $\quad V P_{\text {Rect }}$ es $V p$ de la línea menos la caída de voltaje de dos diodos del puente cuando conducen.

En este circuito, cada etapa de fuente de corriente constante $(C S n)$, conduce entre los ángulos $\alpha_{n} \mathrm{y} \alpha_{n+1}$ hasta llegar a $\pi / 2$ rad, y circulan por cada led, de acuerdo a la conexión en la figura 2. Para calcular la potencia de cada COB-LED se aplica la ecuación 4:

$$
P_{L E D n}=\frac{2 V_{L E D n}}{\pi}\left(\left(\sum_{i=n}^{m-1} I_{C S n}\left(\propto_{(i+1)}-\propto_{i}\right)\right)+I_{C S m}\left(\frac{\pi}{2}-\propto_{m}\right)\right)
$$

Donde $\quad n$ es la etapa de la fuente de corriente y vale $1,2 \ldots$ hasta es el número total de etapas $m$.

Para una sola etapa $(m=1)$, desaparece la sumatoria y $P_{L E D}=\frac{2 V_{L E D 1}}{\pi}\left(I_{C S 1}\left(\frac{\pi}{2}-\propto_{1}\right)\right)$

El valor de $V_{L E D n}$ sigue una ecuación polinómica de segundo orden que puede ser modelada según las especificaciones de los COB-LEDs o los leds que constituyen los arreglos:

$$
V_{\text {Led }}=V_{0}+r_{1} I_{\text {Led }}+r_{2} I_{\text {Led }}^{2}
$$

Donde $\quad V 0$ es el valor de la fuente de voltaje equivalente del led.

$$
r_{1} \text { y } \quad r_{2} \text { representan los coeficientes de }
$$
aproximación cuadrática.
Por ejemplo, para un led Everlight de la serie 62227ET/KK7D, a partir de la curva dada por el fabricante [10] y al hacer la aproximación por el método de mínimos cuadrados, se cumple que: $V 0=2,5729 V ; r_{1}=4,9083 \Omega$ y $r_{2}$ $=-10,5891 \Omega / A$.

Los ángulos de conmutación pueden determinarse de acuerdo a la siguiente ecuación:

$$
\propto_{n}=\operatorname{sen}^{-1}\left(\frac{\sum_{n=1}^{m} V_{L E D n}}{V_{P_{-} R e c t}}\right)
$$

La potencia útil promedio total para todos los leds se calcula con la ecuación 7:

$$
P_{\text {Total_LED }}=\sum_{n=1}^{m} P_{L E D n}
$$

La $P_{\text {Total_LED }}$ es útil para determinar el flujo luminoso de la luminaria y la eficiencia del circuito. Para calcular la eficiencia se necesita la potencia total que consume el circuito de alimentación $P_{C i r}$, la cual se puede determinar para la mitad de un período según la ecuación 8:

$$
P_{\text {Cir }}=\frac{1}{\pi} \int_{0}^{\pi} v_{\text {Cir }}(\theta) i_{\text {Cir }}(\theta) d \theta
$$

Donde $v_{c i r}(\theta)$ es el voltaje de línea de la ecuación $2 \operatorname{con} \theta$ $=\omega t$ :

$$
v_{\operatorname{Cir}}(\theta)=V_{P} \operatorname{sen}(\theta)
$$

La intensidad eléctrica del circuito para un ángulo comprendido desde 0 hasta $\pi / 2$, cuando hay una conmutación perfecta entre cada etapa de fuente de corriente constante es:

$$
i_{C i r}(\theta)=\left.I_{P_{o l P}} \operatorname{Sen}(\theta)\right|_{0} ^{\frac{\pi}{2}}+\left(\left.\sum_{n=1}^{(m-1)} I_{C S 1}\right|_{\alpha_{n}} ^{\alpha_{n+1}}\right)+\left.I_{C S m}\right|_{\alpha_{m}} ^{\frac{\pi}{2}}
$$

El primer término de la ecuación 10 corresponde al consumo aproximadamente resistivo del circuito de polarización para los circuitos de conmutación y de las compuertas de los MOSFETs, los otros términos corresponden a los de cada fuente pulsante de corriente constante (Si m es igual a 1, el término de la sumatoria no se aplica). El consumo de la corriente de polarización es bajo, ya que está constituido por: $R_{4 n-3}(2 \mathrm{M} \Omega), R_{4 n-2}(240$ $\mathrm{k} \Omega), R_{4 n-1}(470 \mathrm{k} \Omega), D_{n}(6,2 \mathrm{~V})$ y $Q_{3 n-2}(2 \mathrm{~N} 3904)$ con $n$ igual 1 , $2,3 \ldots m$, según el número total de etapas.

Para calcular la potencia total del circuito, se aplica la integral de los valores de las corrientes constantes por el voltaje de línea, en cada período de conducción y se suman. También se suma la potencia consumida $P_{\text {pol }}$ de y las pérdidas en el puente rectificador $P_{P 1}$ : 


$$
P_{C i r c}=\frac{2 V_{P}\left[\left(\sum_{n=1}^{(m-1)} I_{C S n} \cos \left(\alpha_{(n+1)}-\alpha_{n}\right)\right)+I_{C S m} \cos \left(\frac{\pi}{2}-\alpha_{m}\right)\right]}{\pi}+P_{P_{o l}}+P_{P 1}
$$

Como la corriente de polarización es aproximadamente sinusoidal, la $P_{p_{o l}}$ promedio es:

$$
P_{\text {Pol }}=\frac{V_{P} \text { Rect } I_{P o l} P}{2}
$$

Al asumir que los voltajes de los diodos rectificadores se mantienen constantes, entonces, la potencia promedio $P_{P 1}$ es:

$$
P_{P 1}=2 V_{a k} I_{R M S_{-} C i r}
$$

$V_{a k}$ es el voltaje de conducción directa en un diodo del puente rectificador, el cual es aproximadamente igual a 0,7 V. La $I_{R M S_{-} \text {Cir }}$ se calcula según la ecuación 14:

$$
I_{R M S_{-} C i r}=\frac{\sqrt{\left(\sum_{n=1}^{(m-1)} I_{C S_{n}}^{2} \cos \left(\alpha_{(n+1)}-\alpha_{n}\right)\right)+I_{C S_{m}}^{2} \cos \left(\frac{\pi}{2}-\alpha_{m}\right)}}{2 \pi}+\frac{I_{P o l P}}{\sqrt{2}}
$$

La eficiencia del circuito se calcula según la siguiente ecuación:

$$
\eta_{\text {Circ }}=\frac{P_{\text {Total } L E D}}{P_{\text {Circ }}} 100 \%
$$

Como no hay desfase entre voltaje y corriente, el factor de potencia se obtiene según:

$$
P F=\frac{P_{\text {Circ }}}{V_{R M S} I_{R M S}}
$$

Por el mismo hecho de no haber desplazamiento de fase, para calcular la distorsión armónica total de la corriente, se puede utilizar la siguiente ecuación [11]:

$$
T H D=100 \% \times \sqrt{\frac{1}{P F^{2}}-1}
$$

Finalmente, para calcular los valores de los armónicos de la corriente, se determinan los armónicos de un solo pulso y para formar la onda completa de la corriente de entrada, se suma cada pulso, con diferente ancho y amplitud. Debido a que la función de la corriente es impar, entonces $a_{n}=0$ para todo $n$. y como el pulso es simétrico con respecto a $\pi / 2$, para cada pulso se cumple que el armónico $b_{n}$ tiene un valor nulo si $n$ es par. Entonces:

$$
b_{(2 n+1)}=\frac{4 I_{k}}{(2 n+1) \pi} \cos \left((2 n+1) \alpha_{k}\right)
$$

Donde $k$, es el índice que presenta el valor de la corriente del pulso y $\alpha_{k}$ el ángulo cuando comienza ese pulso, entonces, la corriente del circuito se puede determinar según la ecuación:

$$
\begin{gathered}
I_{\text {Cir }}=\frac{4 I_{1}}{(2 n+1) \pi} \sum_{n=1}^{\infty} \cos (2 n+1) \alpha_{1}+\frac{4 I_{2}}{(2 n+1) \pi} \sum_{n=1}^{\infty} \cos (2 n+1) \alpha_{2}+\ldots \\
\ldots \frac{4 I_{m}}{(2 n+1) \pi} \sum_{n=1}^{\infty} \cos (2 n+1) \alpha_{m}
\end{gathered}
$$

\section{Parte Experimental}

Para realizar las simulaciones, se utiliza el modelo matemático de arreglos del led Everlight de la serie $62-227 \mathrm{ET} / \mathrm{KK} 7 \mathrm{D}$, ya que ofrecen una buena eficacia: $228 \mathrm{~lm} / \mathrm{W}$ a $25^{\circ} \mathrm{C}$. Las potencias de las fuentes de alimentación serían de $5 \mathrm{~W}$, para un voltaje nominal de $120 \mathrm{~V}$, y de $10 \mathrm{~W}$, para un voltaje nominal de $230 \mathrm{~V}$. Los flujos luminosos que deben producir, son de $1140 \mathrm{y}$ 2280 lúmenes respectivamente. En la tabla 1 se resumen los valores, obtenidos en la simulación, de las fuentes de alimentación de corriente pulsante alimentadas con 120 voltios.

En las figuras 3 a 8 se muestran las gráficas obtenidas de: potencia, eficiencia y distorsión armónica total para las fuentes alimentadas con $120 \mathrm{~V} \pm 10 \mathrm{~V}$ y 230 $\mathrm{V} \pm 10 \mathrm{~V}$.

En las tablas 2 y 3 se presentan los valores obtenidos en las simulaciones de la relación porcentual de los armónicos en fuentes de 120 Vy 230 V respectivamente.

Potencia de Salida vs. Voltaje de línea a 120 V nominal

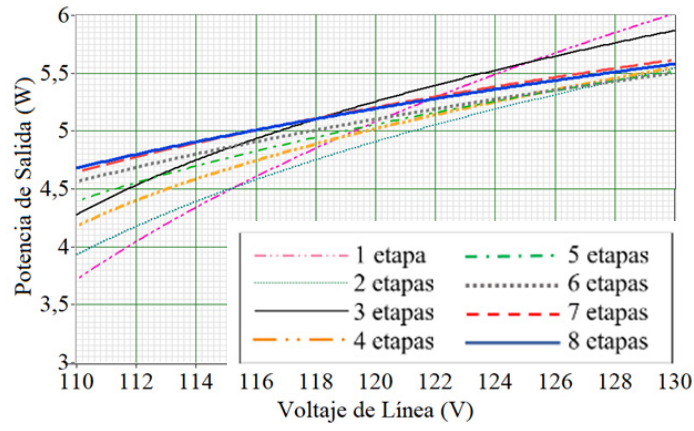

Figura 3. Potencia de salida de fuentes de corriente pulsante de $120 \mathrm{~V}$ para leds.

Potencia de Salida vs. Voltaje de línea a 230 V nominal

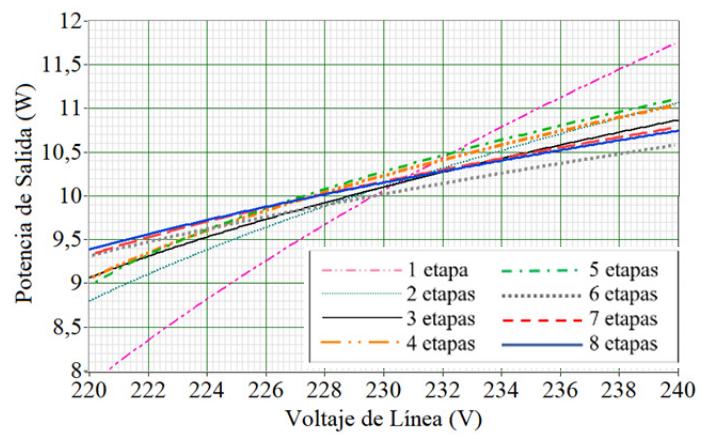

Figura 4. Potencia de salida de fuentes de corriente pulsante de $230 \mathrm{~V}$ para leds. 
Eficiencia vs. Voltaje de línea a 120 V nominal

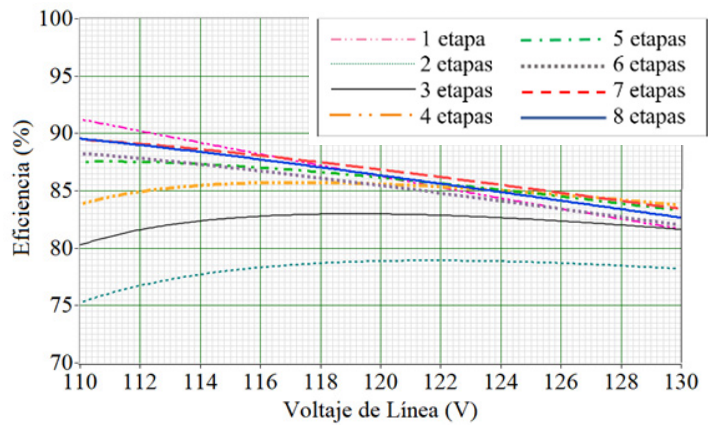

Figura 5. Eficiencia de fuentes de corriente pulsante de $120 \mathrm{~V}$ para leds.

Eficiencia vs. Voltaje de línea a 230 V nominal

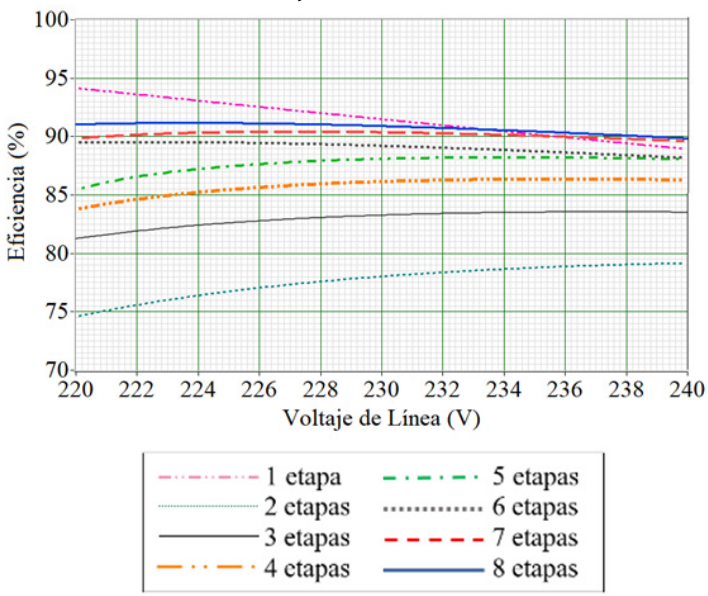

Figura 6. Eficiencia de fuentes de corriente pulsante de $230 \mathrm{~V}$ para leds.

Distorsión Armónica Total vs. Voltaje de línea a 120 V nominal

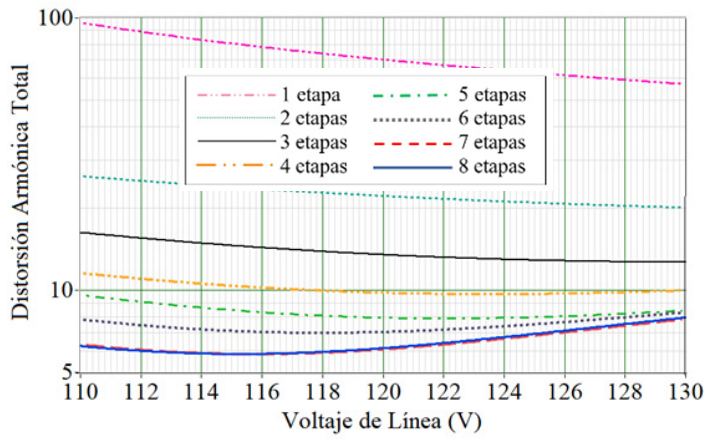

Figura 7. Distorsión Armónica Total en fuentes de corriente pulsante de $120 \mathrm{~V}$ para leds.
Eficiencia vs. Voltaje de línea a 230 V nominal

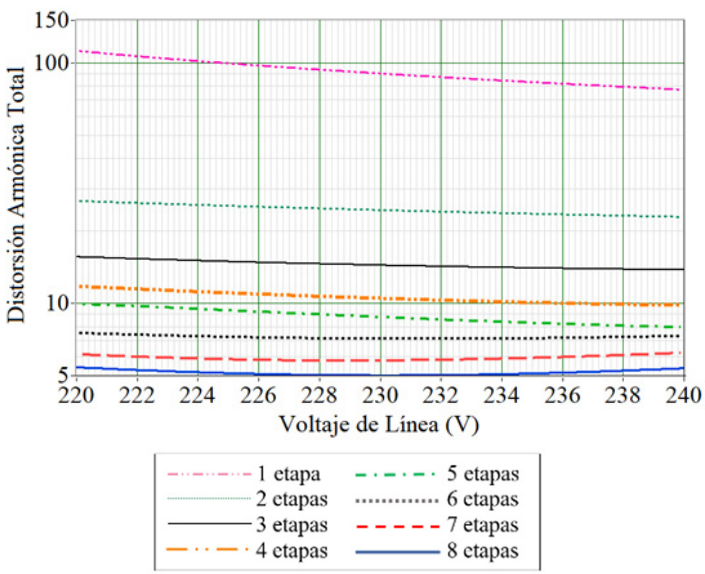

Figura 8. Distorsión Armónica Total en fuentes de corriente pulsante de $230 \mathrm{~V}$ para leds.

Tabla 1. Resumen de los resultados de las simulaciones para las fuentes de alimentación de corriente pulsante de 1 a 8 etapas y un voltaje nominal de $120 \mathrm{~V}$.

\begin{tabular}{|c|c|c|c|c|c|c|c|c|}
\hline $\begin{array}{c}\mathrm{N}^{0} \\
\text { Etapas }\end{array}$ & 1 & 2 & 3 & 4 & 5 & 6 & 7 & 8 \\
\hline $\begin{array}{c}\mathrm{N}^{0} \text { leds } \\
\text { serie por } \\
\text { etapa }\end{array}$ & 46 & 25 & 17 & 13 & 10 & 8 & 7 & 6 \\
\hline $\begin{array}{l}\mathbf{P}_{\mathrm{Sal}} @ \\
120 \mathrm{~V}\end{array}$ & 5,08 & 4,92 & 5,25 & 5,02 & 5,05 & 5,10 & 5,21 & 5,20 \\
\hline$\eta_{120 v}(\%)$ & 86,3 & 79,0 & 83,0 & 85,6 & 86,1 & 85,4 & 86,8 & 86,3 \\
\hline $\begin{array}{c}\text { THD }_{120 \mathrm{~V}} \\
(\%)\end{array}$ & 70,9 & 17,1 & 13,4 & 9,8 & 8,0 & 7,2 & 6,3 & 6,4 \\
\hline $\mathrm{PF}_{120 \mathrm{~V}}$ & 0,818 & 0,976 & 0,991 & 0,995 & 0,997 & 0,998 & 0,998 & 0,998 \\
\hline $\mathbf{P}_{\text {Led } 1}(\mathbf{W})$ & 5,08 & 3,17 & 2,24 & 1,58 & 1,22 & 1,00 & 0,88 & 0,75 \\
\hline $\mathbf{P}_{\mathrm{Led} 2}(\mathbf{W})$ & - & 1,75 & 1,91 & 1,47 & 1,17 & 0,97 & 0,86 & 0,74 \\
\hline $\mathbf{P}_{\text {Led3 }}(\mathbf{W})$ & - & - & 1,10 & 1,24 & 1,08 & 0,93 & 0,83 & 0,73 \\
\hline $\mathbf{P}_{\text {Led } 4}(\mathbf{W})$ & - & - & - & 0,73 & 0,93 & 0,86 & 0,79 & 0,70 \\
\hline $\mathbf{P}_{\text {Led5 }}(\mathbf{W})$ & - & - & - & - & 0,65 & 0,76 & 0,73 & 0,67 \\
\hline $\mathbf{P}_{\text {Led } 6}(\mathbf{W})$ & - & - & - & - & - & 0,58 & 0,63 & 0,62 \\
\hline $\mathbf{P}_{\text {Led } 7}(\mathbf{W})$ & - & - & - & - & - & - & 0,49 & 0,55 \\
\hline $\mathbf{P}_{\mathrm{Led} 8}(\mathrm{~W})$ & - & - & - & - & - & - & - & 0,44 \\
\hline $\mathbf{R}_{4}(\Omega)$ & 6,8 & 13 & 20 & 30 & 39 & 51 & 56 & 68 \\
\hline $\mathbf{R}_{8}(\Omega)$ & - & 9,1 & 12 & 18 & 24 & 30 & 33 & 39 \\
\hline$R_{12}(\Omega)$ & - & - & 9,1 & 12 & 16 & 20 & 24 & 27 \\
\hline $\mathrm{R}_{16}(\Omega)$ & - & - & - & 10 & 13 & 16 & 18 & 22 \\
\hline$R_{20}(\Omega)$ & - & - & - & - & 10 & 13 & 15 & 18 \\
\hline $\mathbf{R}_{24}(\Omega)$ & - & - & - & - & - & 10 & 12 & 15 \\
\hline$R_{28}(\Omega)$ & - & - & - & - & - & - & 10 & 12 \\
\hline $\mathbf{R}_{32}(\Omega)$ & - & - & - & - & - & - & - & 10 \\
\hline
\end{tabular}


Tabla 2. Relación porcentual de armónicos con respecto a la fundamental a $120 \mathrm{~V}$

\begin{tabular}{|c|c|c|c|c|c|c|c|c|c|}
\hline Total de Etapas & 1 & 2 & 3 & 4 & 5 & 6 & 7 & 8 & EN61000 -3-2 \\
\hline PF & 0,818 & 0,976 & 0,991 & 0,995 & 0,997 & 0,998 & 0,998 & 0,998 & \\
\hline Total de leds & 46 & 50 & 51 & 52 & 50 & 48 & 49 & 48 & Clase C \\
\hline \multicolumn{10}{|l|}{$N^{0}$ Armónico } \\
\hline$|\mathbf{b} 3|(\%)$ & 59 & 4,2 & 0,8 & 0,6 & 0,6 & 1,1 & 1,6 & 1,3 & $30 \times$ PF \\
\hline$|\mathrm{b5}|(\mathbf{\%})$ & 7,8 & 6,5 & 2,2 & 2,7 & 1,4 & 2,6 & 2,7 & 3,4 & 10 \\
\hline$|\mathrm{b} 7|(\%)$ & 21,1 & 9,0 & 3,6 & 1,1 & 0,7 & 1,9 & 2,0 & 2,6 & 7 \\
\hline$|\mathrm{b} 9|(\mathbf{0})$ & 17,0 & 11,5 & 5,6 & 1,9 & 3,0 & 1,8 & 0,8 & 0,1 & 5 \\
\hline $\mid$ b11| (\%) & 3,2 & 0,8 & 0,3 & 0,6 & 0,2 & 1,8 & 1,2 & 1,6 & 3 \\
\hline$|\mathrm{b} 13|(\%)$ & 13,8 & 7,3 & 3,0 & 3,5 & 0,5 & 0,8 & 0,4 & 0,0 & 3 \\
\hline$|\mathrm{b} 15|(\%)$ & 7,3 & 3,5 & 6,2 & 3,0 & 2,7 & 0,8 & 0,5 & 0,4 & 3 \\
\hline |b17| (\%) & 6,9 & 3,2 & 0,2 & 1,2 & 1,2 & 1,4 & 0,5 & 0,9 & 3 \\
\hline$|\mathrm{b} 19|(\%)$ & 9,8 & 0,5 & 3,1 & 1,1 & 0,2 & 0,4 & 0,2 & 0,0 & 3 \\
\hline$|\mathrm{b} 21|(\%)$ & 2,0 & 5,4 & 2,8 & 3,8 & 2,6 & 0,7 & 1,4 & 0,4 & 3 \\
\hline$|\mathbf{b} 23|(\%)$ & 6,3 & 3,6 & 3,7 & 1,2 & 1,0 & 2,1 & 0,8 & 0,8 & 3 \\
\hline$|\mathbf{b} 25|(\%)$ & 6,1 & 0,4 & 0,8 & 1,1 & 1,5 & 0,0 & 0,6 & 0,2 & 3 \\
\hline |b27| (\%) & 1,1 & 0,7 & 1,6 & 2,6 & 0,9 & 0,4 & 0,6 & 0,1 & 3 \\
\hline$|\mathbf{b} 29|(\%)$ & 6,0 & 2,8 & 0,6 & 2,8 & 2,3 & 1,7 & 1,5 & 1,2 & 3 \\
\hline$|\mathrm{b} 31|(\%)$ & 3,8 & 3,4 & 0,1 & 0,4 & 0,7 & 0,9 & 0,4 & 0,2 & 3 \\
\hline$|\mathbf{b 3 3}|(\%)$ & 5,3 & 0,8 & 2,0 & 0,3 & 0,0 & 1,2 & 0,2 & 0,5 & 3 \\
\hline$|\mathbf{b 3 5}|(\%)$ & 0,7 & 2,2 & 1,6 & 1,4 & 1,2 & 0,1 & 1,6 & 1,0 & 3 \\
\hline$|\mathbf{b 3 7}|(\%)$ & 3,8 & 1,4 & 1,8 & 0,4 & 2,6 & 1,5 & 0,8 & 1,0 & 3 \\
\hline$|\mathrm{b39}|(\%)$ & 1,2 & 1,8 & 0,9 & 1,4 & 1,0 & 1,0 & 1,0 & 0,8 & 3 \\
\hline
\end{tabular}

Tabla 3. Relación porcentual de armónicos con respecto a la fundamental a $230 \mathrm{~V}$

\begin{tabular}{|c|c|c|c|c|c|c|c|c|c|}
\hline Total de Etapas & 1 & 2 & 3 & 4 & 5 & 6 & 7 & 8 & EN61000 \\
\hline $\mathbf{P F}$ & 0,742 & 0,971 & 0,990 & 0,995 & 0,996 & 0,997 & 0,998 & 0,999 & $3-2$ \\
\hline Total de leds & 92 & 100 & 102 & 104 & 105 & 102 & 105 & 104 & Clase C \\
\hline \multicolumn{10}{|l|}{$N^{0}$ Armónico } \\
\hline$\left|b_{3}\right|(\%)$ & 68,8 & 3,9 & 0,2 & 0,4 & 1,5 & 0,2 & 1,6 & 0,1 & $30 \times \mathrm{PF}$ \\
\hline$\left|\mathbf{b}_{5}\right|(\%)$ & 24,0 & 7,3 & 4,4 & 1,3 & 0,4 & 2,7 & 1,6 & 1,9 & 10 \\
\hline$\left|\mathbf{b}_{7}\right|(\mathbf{\%})$ & 11,3 & 13,2 & 5,4 & 2,6 & 1,4 & 1,6 & 0,2 & 0,8 & 7 \\
\hline$\left|\mathbf{b}_{9}\right|(\%)$ & 23,0 & 9,5 & 2,8 & 1,4 & 1,2 & 0,3 & 0,3 & 0,3 & 5 \\
\hline$\left|b_{11}\right|(\%)$ & 12,5 & 5,0 & 0,0 & 1,2 & 1,6 & 1,5 & 0,0 & 0,0 & 3 \\
\hline$\left|b_{13}\right|(\%)$ & 4,4 & 6,1 & 5,8 & 4,9 & 3,2 & 1,6 & 0,9 & 0,7 & 3 \\
\hline$\left|b_{15}\right|(\%)$ & 13,2 & 4,3 & 4,8 & 0,2 & 1,3 & 0,2 & 0,3 & 0,2 & 3 \\
\hline$\left|b_{17}\right|(\%)$ & 9,0 & 2,8 & 1,4 & 0,4 & 1,2 & 0,7 & 1,3 & 0,6 & 3 \\
\hline$\left|b_{19}\right|(\%)$ & 1,9 & 4,4 & 3,3 & 3,7 & 3,0 & 2,2 & 1,2 & 1,1 & 3 \\
\hline$\left|b_{21}\right|(\%)$ & 9,1 & 4,9 & 4,4 & 2,3 & 0,8 & 0,2 & 0,4 & 0,0 & 3 \\
\hline$\left|b_{23}\right|(\%)$ & 7,3 & 1,0 & 1,9 & 0,4 & 0,2 & 0,7 & 1,2 & 1,0 & 3 \\
\hline$\left|b_{25}\right|(\%)$ & 0,5 & 0,0 & 1,4 & 2,5 & 1,6 & 2,2 & 0,8 & 0,7 & 3 \\
\hline
\end{tabular}




\begin{tabular}{|c|c|c|c|c|c|c|c|c|c|}
\hline$\left|\mathbf{b}_{27}\right|(\%)$ & 6,7 & 3,4 & 0,4 & 3,5 & 2,2 & 1,4 & 0,6 & 0,8 & 3 \\
\hline$\left|b_{29}\right|(\%)$ & 6,2 & 3,6 & 0,6 & 0,5 & 1,0 & 1,0 & 1,2 & 1,0 & 3 \\
\hline$\left|b_{31}\right|(\%)$ & 0,4 & 0,6 & 1,3 & 1,0 & 2,5 & 0,3 & 1,6 & 1,0 & 3 \\
\hline$\left|b_{33}\right|(\%)$ & 5,1 & 1,6 & 1,8 & 1,3 & 1,4 & 1,4 & 0,0 & 0,7 & 3 \\
\hline$\left|b_{35}\right|(\%)$ & 5,4 & 2,3 & 2,7 & 0,7 & 1,0 & 1,7 & 0,8 & 0,2 & 3 \\
\hline$\left|\mathbf{b}_{37}\right|(\%)$ & 0,9 & 2,2 & 0,8 & 0,7 & 2,1 & 0,1 & 0,0 & 1,4 & 3 \\
\hline$\left|b_{39}\right|(\%)$ & 4,0 & 1,5 & 2,3 & 0,8 & 0,3 & 1,4 & 1,6 & 0,7 & 3 \\
\hline
\end{tabular}

En la tabla 4, se pueden observar lo resultados obtenidos de autores y fabricantes, en las que se utilizan otros tipos de fuentes de alimentación para lámparas leds.

\section{Resultados y Discusión}

De acuerdo a los resultados obtenidos en

Tabla 4. Cuadro comparativo entre varias fuentes de alimentación para leds.

\begin{tabular}{ccccccccc}
\hline Ref & Método & Año & Autor o Fabricante & $\eta(\%)$ & PF & C & L & Voltaje (V) \\
\hline$[12]$ & Conmutación & 2010 & Strato & 81 & 0,96 & Sí & Sí & 90 a 270 \\
{$[12]$} & Reactancia & 2010 & Diversos & 93 & 0,33 & Sí & No & 120 \\
{$[12]$} & I pulsante, 1 etapa & 2012 & Universidad del Zulia & 91 & 0,87 & No & No & 120 \\
{$[12]$} & Conmutación & 2014 & LinkSwitch & 82 & 0,90 & Sí & Sí & 90 a 270 \\
{$[13]$} & Conmutación & 2012 & Philips Hue RGB & 74 & 0,49 & Sí & Sí & 120 \\
{$[13]$} & Conmutación & 2014 & Cree Connected & 84 & 0,99 & Sí & Sí & 120 \\
{$[13]$} & Conmutación & 2014 & GE Link LED & 85 & 0,94 & Sí & Sí & 120 \\
{$[13]$} & Conmutación & 2014 & LG Smart Lamp & 77 & 0,54 & Sí & Sí & 120 \\
{$[13]$} & Capacitor conmutado & 2016 & Ko, et al & 78 & 0,72 & Sí & No & 120 \\
{$[14]$} & I pulsante, 3 etapas & 2011 & Hwu, et al & 85 & 0,97 & No & No & 120 \\
{$[15]$} & I pulsante, 4 etapas & 2015 & Acrich 3 Seoul Sem & 86 & 0,97 & No & No & 120 y 220 \\
{$[16]$} & I pulsante, 4 etapas & 2017 & CL88020 Microchip & 85 & 0,95 & No & No & 120 y 230 \\
{$[17]$} & I pulsante, 6 etapas & 2015 & CL8800, Microchip & 85 & 0,95 & No & No & 120 y 230 \\
- & I pulsante, 8 etapas & 2018 & Este trabajo & 87 & 1,00 & No & No & 120 \\
\hline & I pulsante, 8 etapas & 2018 & Este trabajo & 91 & 1,00 & No & No & 230 \\
\hline
\end{tabular}

las gráficas y las tablas, se puede afirmar que, las características de eficiencia, factor de potencia y distorsión armónica total, mejoran al aumentar el número de etapas, con excepción para el caso de 8 etapas en 120 $\mathrm{V}$, en donde no se nota una mejora con respecto al de 7 etapas. El circuito de una sola etapa es el que presenta el menor factor de potencia y más distorsión armónica total, pero mantiene una alta eficiencia.

El mejor resultado se obtiene para el caso de 8 etapas y $230 \mathrm{~V}$, donde, el factor de potencia es casi igual a 1, la eficiencia 91\% y la distorsión armónica total 5\%. En la tabla 4, se puede comparar este circuito con respecto a otros ya publicados. Según estos resultados, con los leds propuestos, se puede obtener una eficacia total de 207 $\mathrm{lm} / \mathrm{W}(228 \mathrm{~lm} / \mathrm{W}$ X 0,909).
El circuito de 2 etapas es el que presenta la más baja eficiencia (78\%) y un factor de potencia de 0,97. Para el de 3 etapas el factor de potencia es 0,99 , con $83 \%$ de eficiencia y, para el de 4 etapas el factor de potencia es 0,995 y una eficiencia de aproximadamente 86\%. De acuerdo a las tablas 2 y 3 , ninguno de los circuitos de 1 a 4 etapas cumplen cabalmente con la norma EN 61000-3-2 clase $\mathrm{C}$, porque hay armónicos que rebasan el límite que fija la norma.

Las fuentes de alimentación de 5 a 8 etapas, cumplen con la norma EN 61000-3-2 clase C y tienen mejores características de factor de potencia, distorsión armónica total y eficiencia. Esto refleja que, a mayor número de etapas, se obtienen mejores características, pero se eleva el número de componentes que utiliza la fuente de alimentación. 
En cuanto a la estabilidad en la potencia de salida, se observa que, para la fuente de una etapa, hay una variación de $\pm 25 \%$, cuando el voltaje de entrada de $120 \mathrm{~V}$ varía $\pm 10 \mathrm{~V}$; en cambio, para los de 7 y 8 etapas esta variación es de $\pm 8,7 \%$. En el caso de la alimentación de $230 \mathrm{~V}$, al variar la entrada en $\pm 10 \mathrm{~V}$, la variación de potencia es de $\pm 20 \%$ para la fuente de 1 etapa $\mathrm{y} \pm 6,5 \%$ para 8 etapas. Esto indica que hay más estabilidad a la salida, a mayor número de etapas. Una solución para reducir estas variaciones y controlar la potencia de salida, consiste en colocar una etapa de compensación y sustituir los transistores bipolares que detectan la corriente según la figura 2, por amplificadores operacionales, como se muestra en la figura 9.
Como la fuente de alimentación descrita en la figura 2, no utiliza inductores ni condensadores, presenta un parpadeo con una frecuencia igual al doble de la que hay en la línea de alimentación eléctrica. Este efecto es imperceptible para la mayoría de las personas. Sin embargo, puede causar problemas cuando la luz de este circuito se utilice en fotografía o filmación digital. Una solución consiste en colocar difusores de material fosforescente en la luminaria, de manera de filtrar las variaciones de intensidad lumínica [18]. Otra solución consiste en conectar tres circuitos iguales con alimentación trifásica; con ello, se produciría un efecto de "rectificación lumínica" de 6 ondas, que prácticamente anulan el efecto de parpadeo.

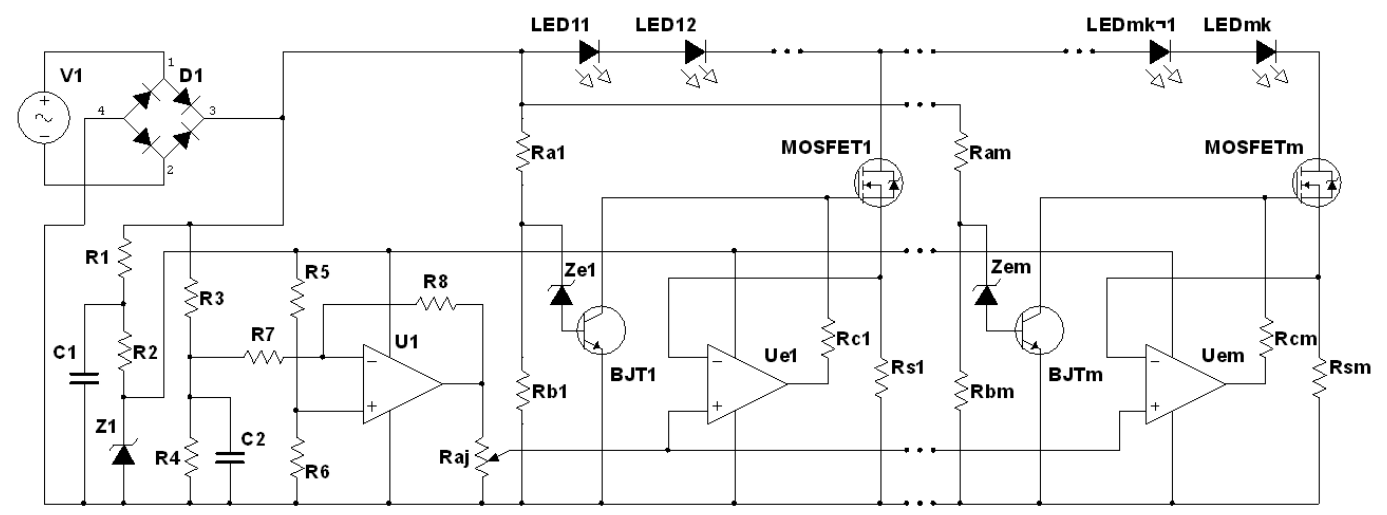

Figura 9. Circuito para la compensación y regulación de la potencia de salida.

$$
V_{o 1}=V_{z} 1 \frac{R 5(R 7+R 8)}{(R 5+R 6) R 7}-V_{P_{-} l i ́ n e a} \frac{R 4 R 8}{(R 3+R 4) R 7}
$$

La resistencia Raj, puede controlar simultáneamente el nivel de corriente de cada etapa por medio de Vo1, el cual tiene un coeficiente negativo con respecto al voltaje de línea. En este punto, también se puede colocar la salida analógica de un microcontrolador con acceso a una red inalámbrica, como aplicación del Internet de las cosas (IoT).

Si se desea obtener una alta eficiencia en el circuito, el valor de Vo1 debe ser menor a 0,5 V cuando la posición de $R a j$ esté al $100 \%$. Como la variación de potencia es bastante lineal, la regulación de potencia se hace a lazo abierto. Para mantener la potencia constante, el segundo término de la ecuación 20 debe ser tal, que Vo1 se reduzca en el mismo porcentaje con el que aumentaría la potencia debido al incremento de $V P_{\text {_línea }}$. Por ejemplo, si hay una variación de 10 Vrms en el voltaje de línea en la fuente de 8 etapas y $120 \mathrm{~V}$, el porcentaje de cambio de Vo1 debe ser, según la figura 3, un 8,7\%; de esta manera se estabilizaría la potencia de salida.

\section{Conclusiones}

En este artículo se analizan circuitos multietapas de corriente pulsante constante para la alimentación de leds con aplicaciones en iluminación en general, los cuales presentan ventajas con respecto a las fuentes conmutadas, tales como simplicidad, bajo costo y larga vida útil.

En los circuitos analizados, al aumentar el número de etapas de corriente constante, aumenta la eficiencia y el factor de potencia, se reduce la distorsión armónica total y se mejora la estabilidad ante variaciones del voltaje de alimentación. Para mantener el flujo luminoso promedio constante, se presenta una propuesta del circuito alternativo de la figura 9.

Para cumplir con la norma IEC61000-3-2 clase $C$, la cual es referida a los equipos de iluminación, es suficiente la utilización de circuitos de 5 o más etapas. Los circuitos de 8 etapas pueden utilizarse con buen desempeño y pueden acoplarse a un gran número de modelos de COB-LEDs de 18 o 36 voltios ofrecidos por muchos de los fabricantes de leds para iluminación. 


\section{Referencias Bibliográficas}

[1] Acevedo R., Araujo E. y Carvajal E. "Diseño y Construcción de una Lámpara Basada en LED para Iluminación Residencial". Trabajo de Grado para optar al título de Ingeniero Electricista. Universidad del Zulia, Maracaibo, Venezuela (2010).

[2] Brito Y. "Desarrollo de un Sistema de Iluminación Led para una Oficina de la Universidad del Zulia". Trabajo de Grado para optar al título de Ingeniero Electricista. Universidad del Zulia, Maracaibo, Venezuela (2012).

[3] Bender V., Marchesan T. and Alonso M. "Solid State Lighting, a Concise Review of State of the art on LED and OLED Modeling". IEEE Industrial Electronics Magazine, June 2015, pp 6 - 16. USA.

[4] Scholand M. and Dillon H. "Life Cicle Assessment of Energy and Environmental Impacts of LED Lighting Products, Part 2: LED Manufacturing and Performance". U.S. Department of Energy (2012).

[5] Tsorng-Juu L., Wei-Jing T., Jiann-Fuh C. and JyunPeng W. "A Novel Line Frequency Multistage Conduction LED Driver with High Power Factor". IEEE Transactions on Power Electronics. Vol. 30. № 9, pp 5103 - 5115. USA (2015).

[6] Gu L., Ruan X., Xu M. and Yao, K. "Means of Eliminating Electrolytic Capacitor in AC/DC Power Supplies for LED Lightings". IEEE Transactions on Power Electronics, Vol 4, No 5, pp 1399 - 1408. May 2009. USA.

[7] Feng W., He Y., and Shi F. "Investigation of LED Light Output Performance Characteristics Under Different Alternating Regulation Modes". IEEE Journal of Selected Topics in Quantum Electronics, Vol 7 No. 3, May/June 2011. USA.

[8] Lee E., Nguyen D. and Rim C. "A Novel Passive type LED Driver for Static LED Power Regulation by Multi-Stage Switching Circuits" IEEE Applied Power Electronics Conference and Exposition (APEC), pp 900 - 905. USA (2015).

[9] Kim J., Lee J. and Park S. "A Soft Self-Commutating Method Using Minimun Control Circuitry for Multiple-String LED Drivers". IEEE International Solid-State Conference, pp 376 - 378. USA, 2013
KK7D-3MXXXXXXX2629U6/2T/EU”. Data Sheet. Taiwan. Disponible en: https://everlightamericas. com/2204/white-lighting-leds. Consultado en diciembre de 2017.

[11] Mohan N., Undeland T. y Robbins W. "Electrónica de Potencia Convertidores, Aplicaciones y Diseño". Mc Graw Hill, 3a Edición. México (2011).

[12] Álvarez C. "Factibilidad Técnica, Económica y Ambiental para la Aplicación de los Sistemas de Iluminación Led en Venezuela". Universidad del Zulia, Venezuela (2015).

[13] Younghun, K., Hong-Soo C., Sang-Sung L., So-Bong S., Yongchull S. and Sang-Guu L. "A Compact FlickerFree Transformer-Less LED Driver with Enhanced Power Factor for Omnidirectional Multichannel Smart Bulb Applications". IEEE Transactions on Power Electronics, Vol. 31, N 8, August 2016. USA.

[14] Hwu K. and Tu W. "A Brightness Light-Emitting Diode Driver with Power Factor and Total Harmonic Distortion Improved". 2011 26th Annual IEEE Applied Power Electronics Conference and Exposition. USA.

[15] Seoul Semiconductor. "Acrich IC 3". Acrich Deveploment, South Korea. Disponible en: http:// npola.cn/AcrichNewWave/Acrich3\%20IC_Rev3.pdf (2014). Consultado en noviembre de 2017.

[16] Microchip. "CL88020 Sequential Linear LED Driver”. USA (2017). Disponible en: http://ww1.microchip. com/downloads/en/DeviceDoc/20005753A.pdf. Consultado en septiembre de 2018.

[17] Microchip. "CL8800 Sequential Linear LED Driver". USA (2015). Disponible en: http://ww1.microchip. com/downloads/en/DeviceDoc/20005357A.pdf. Consultado en septiembre de 2018.

[18] Tan J. and Narendran N. "Defining Phosphor Luminescence Property Requirements for White AC LED Flicker Reduction". Science Direct, Journal of Luminescence, pp 21 - 26. 2015.

[10] Everlight. "SMD MID POWER LED 62-227ET/ 


\section{REVISTA TECNICA}

DE LA FACULTAD DE INGENIERIA

UNIVERSIDAD DEL ZULIA

Vol. 42. N², Mayo -Agosto 2019, pp. 47 - 97

Esta revista fue editada en formato digital y publicada

en Abril de 2019, por el Fondo Editorial Serbiluz, Universidad del Zulia. Maracaibo-Venezuela

www.luz.edu.ve

www.serbi.luz.edu.ve

produccioncientifica.luz.edu.ve 